\title{
Comparison of Heart Rate Variability between Shift and Rest Days in Nurses Working at Coronary Intensive Care Unit
}

Mehmet Ozgeyik', [MD]

ORCID: 0000-0002-8510-3505

Taner Sen ${ }^{2}$, [MD]

ORCID: 0000-0003-2464-2457

Mehmet Ali Astarcioglu², [MD]

ORCID: 0000-0001-8577-9890

Mufide Okay ${ }^{3}$, [MD]

ORCID: 0000-0001-5317-0597

Abdulkadir Uslu 4 , [MD]

ORCID: 0000-0002-3022-2734

'Eskisehir City Hospital, Department of Cardiology, Eskisehir, Turkey.

${ }^{2}$ Kutahya Dumlupinar University, Department of Cardiology, Kutahya, Turkey.

${ }^{3}$ Eskisehir City Hospital, Department of Hematology, Eskisehir, Turkey.

${ }^{4}$ Kosuyolu Research and Training Hospital, Department of Cardiology, Istanbul, Turkey.

Corresponding Author: Mehmet Ozgeyik Department of Cardiology, Eskisehir City Hospital, Eskisehir, Turkey.

E-mail: mehmetozgeyik@hotmail.com

\section{w ABSTRACT Con}

Objective: Stress of working conditions change the circadian rhythm and body's autonomic functions. Heart rate variability is a simple and noninvasive method that reflects the heart's autonomic system. In this study, we analyzed the rhythm differences of intensive care unit nurses between 24 hours shift and rest days.

Materials and Methods: 51 nurses working in intensive care units of our center were included in the study. The nurses were divided into two groups as study and control groups according to the shift and rest days for 24 hours holter recordings, respectively.

Results: Heart rate parameters were under parasympathic and sympathic dominance in rest and shift days, respectively. Although normalized high frequency, normalized low frequency and low frequency/high frequency ratio values were parallel to the shift and rest days at 24hour intervals, inverse correlations were observed between 03.00- 08.00 hours. No statistically significant difference was seen in circadian rhythm between shift and rest days. Heart rate parameters generally showed similar pattern of circadian rhythm irrespective of the working status of the participants. Sympathetic activity was significantly higher in the day period than the night period in the shift days however there was no difference within resting group.

Conclusion: Slight increase in sympathetic system activity and marked decrease in parasympathetic activity during 03-05 o'clock were apparent in shift days. This increase was attributed to early cortisol release.

Keywords: Work shift, 12-lead electrocardiogram, health-care staff, heart rate variability

https://doi.org/10.32552/2021.ActaMedica.531

Received: 3 November 2020, Accepted: 16 December 2020

Published online: 5 February 2021

\section{INTRODUCTION}

The effects of mental and physical stresses associated with working conditions of health personnel have been evaluated in various studies over the last decade. Mental and physical stress of working conditions change the circadian rhythm of the body. Autonomic functions of the heart are also affected by these changes. Fluctuations of the autonomic status may increase the cardiovascular risk of shifting health workers [1-3]. In addition, perceived work stress has been shown to be associated with coronary heart disease in many studies $[4,5]$. Health personnel working in emergency departments, such as intensive care, are exposed to more stress than medical personnel in the non-emergency departments.

Previous studies showed that heart rate and blood pressure are increased during working hours, and decreased to normal levels in non-working hours $[6,7]$. The resting heart rate is determined by both sympathetic and parasympathetic system 
[8]. Increased sympathetic activity leads to life threatening arrhythmias by reducing ventricular refractory period, promoted triggered activity and enhanced automaticity. Heart rate variability (HRV) is a simple and noninvasive method that reflects the autonomic system of the heart $[9,10]$. HRV is based on the principle of measuring heart rate variation from beat to beat [11]. Ito et al. showed that the HRV of the shifting nurses was affected by physical activity independent of working hours [12]. Another study designed by Siliskovic et al. showed that there is a mismatch between the upper work limits of the body and the working requirements in the night shifts due to parasympathetic dominance which is more dominant at nights. They also showed that early morning hours were the period of lowest arousal and this is the reason of many work related mistakes might occur in this time period [13].

In this study, we analyzed the rhythm differences of intensive care unit nurses between 24 hours shift and rest days to examine whether the circadian rhythm and HRV variables differ between working and rest days.

\section{MATERIALS and METHODS}

\section{Study Population}

51 nurses working for 24-hour shifts in intensive care units of our center were included in the study. The working conditions of the intensive care unit at the shift days were similar for all nurses. On resting days, nurses were told to sleep only at night time and perform their daily activities normally. Demographic data and recordings were evaluated in the study. Demographic data were age, gender, height, weight, marital status, smoking and total working time in intensive care unit. A written consent form was signed by the participants. The conduct of this study was approved by the Institutional Ethics Committee (G-271/80558721). This study was performed in intensive care units between September 2019 and December 2019. The study was designed in accordance with the Helsinki Declaration's ethical standards. Exclusion criteria were history of any cardiac diseases, hypertension and diabetes mellitus. Nurses with a history of drug use affecting the heart rate and heart rhythm were excluded from the study. Those who have a history of alcohol use were also excluded from the study.

\section{ECG Monitoring and HRV Analysis}

The nurses were divided into two groups as study and control groups according to the shift and rest days, respectively. The rest days were selected as 48 hours after working days in order to reduce the effect of sleep deprivation. A 24-hour ECG Holter device (3 channels Beneware Cardiotrak CT-08, China) was placed to all nurses during their 24-hours shift days and rest days (09.00-09.00). Data were analyzed using the same brand's software. Artifacts and rhythm disturbances during recording were evaluated by the same physician and excluded from the HRV analysis. Recordings shorter than under 23 hours were not included in the study and holter recordings were repeated under the same conditions ( 3 nurses at rest days, 5 nurses at shift days). For all recordings, any rhythm disturbance and ischemic findings were not observed. From holter recordings; maximum heart rate $(H R)$, average $H R$, minimum $H R$, supra ventricular and ventricular extra pulses, 24-hour time domain parameters and frequency domain parameters were obtained. NN value is a time interval between 2 consecutive heart beats. Time domain parameters are mean NN, standard deviation of NN interval (SDNN), standard deviation of the average of NN intervals in 5-min segments (SDANN), square root of the mean squared differences of successive NN intervals (rMSSD), proportion derived by dividing NN50 by the total number of NN intervals (pNN50). Frequency domain parameters are total power (TP), very low frequency power (VLF), low frequency power (LF), high frequency power (HF), normalized low frequency (LFnu) and normalized high frequency (HFnu) and LF/HF ratio.

LF parameter reflects the combined effects of both parasympathetic and sympathetic system activity. HF parameter reflects modulation of efferent parasympathetic activity by ventilation. LFnu and HFnu shows the influence of sympathetic and parasympathetic activity on HRV respectively.

\section{Statistical Analysis}

Descriptive statistical results of the study data were expressed as mean \pm standard deviation. The normal distribution of data was examined by Kolmogorov-Smirnov test. Parametric tests (Paired Samples T test) were used for normal distribution and non-parametric tests (Wilcoxon Signed Ranks test) were used for abnormal distribution. Pearson's Chi-Square and Pearson Correlation tests were used 
for the analysis of categorical and numerical data. $P$ values of less than 0.05 were considered significant in the results obtained from the statistical tests. Data were analyzed using SPSS 16.0 (Statistical Package for the Social Sciences for Windows, Chicago, Illinois).

\section{RESULTS}

In this study, a total of 51 nurses, (15 males and 36 females) constitute the study population. The mean age of the study group was $29.35 \pm 6.408$. $37.3 \%$ of the study group were active smoker and $49 \%$ of the study group were married. The other characteristics of the group were shown in Table 1. The mean duration of working in intensive care unit was 39.12 (3-130) months. There was a statistical difference between mean $H R$, minimum $H R$, 24hour average $\mathrm{NN}$, daytime mean $\mathrm{NN}$ and night time mean NN parameters at rest and shift days $(p=0.177, p=0.002, p=0.000, p=0.002, p=0.000$, respectively). Average HR and minimum HR were higher in shift days, however 24-hour average NN, day average $\mathrm{NN}$ and night average $\mathrm{NN}$ values were higher at rest days (Table 2). There was no statistical difference between rest and shift days according to the maximum HR $(p=0.177)$.

Table 1. Descriptive characteristics of the study group.

\begin{tabular}{|l|c|}
\hline Variables & $\mathrm{n}(\%)(\mathrm{n}=51)$ \\
\hline Age (year) (mean \pm SD) & $29,35 \pm 6,408(21-44)$ \\
Gender (Female) & $36(70.6 \%)$ \\
Smoker & $19(37.3 \%)$ \\
Body Mass Index & \\
(mean \pm SD) (Range) & $24.79 \pm 4.08(19.03-38.28)$ \\
Marital status & $25(49 \%)$ \\
Single & $39.12 \pm 32.89(3-130)$ \\
\hline IC working time (months)
\end{tabular}

*SD: standard deviation, M: male, F: female, IC: intensive care
When compared the 24-hour HRV parameters; SDNN, SDANN, rMSSD, pNN50, TP, VLF, HF, LFnu, HFnu and LF/HF ratio parameters were statistically significant different between rest and shift days' groups $(p=0.00, p=0.00, p=0.00, p=0.002, p=0.04$, $p=0.017, p=0.001 p=0.000, p=0.000, p=0.000$, respectively). SDNN, SDANN, rMSSD, pNN50, TP, $\mathrm{VLF}, \mathrm{HF}$ and HFnu values were higher for the rest day group, whereas LFnu and LF/HF ratios were higher for shift day group (Table 3).

When daytime HRV parameters were analyzed; SDNN, SDANN, rMMSD, pNN50, TP, HF, LFnu, HFnu and LF/HF ratio were statistically significant different between the rest and shift days' groups ( $p=0.001, p=0.001, p=0.001, p=0.002, p=0.014$, $p=0.001, p=0.014, p=0.001, p=0.035$, respectively). The values of SDNN, SDANN, rMSSD, pNN550, TP, $\mathrm{HF}$, HFnu were higher at rest days, however LFnu and LF / HF values were higher at shift days. When night time HRV parameters were analyzed; SDANN, rMMSD, pNN50, LFnu, HFnu and LF/HF ratios were statistically significant different between night time rest and shift days' groups $(p=0.053$, $p=0.005, p=0.002, p=0.001, p=0.001$ and $p=0.000$, respectively). rMSSD, pNN50 and HFnu values were higher at rest days; however, SDANN, LFnu and LF / $\mathrm{HF}$ ratios were higher at shift days.

Rest and shift days' mean NN values were parallel to each other for 24 hours follow-up. However, there was a sudden brief drop of mean NN value at night shifts when the nurses were nearly finished their shift in the early morning (Figure 1).

24-hours trend of SDNN parameter generally showed similar pattern between shift and rest days but it had sharp increase during 04:00-07:00 time period in rest days while slight decrease in shift days in the same time period (Figure 2).

Table 2. Comparison of heart rate parameters at rest and shift days groups.

\begin{tabular}{|l|c|c|c|}
\hline Heart rate parameters $(\mathrm{bpm})$ & Rest & Shift (Work) & $\mathrm{p}$ \\
\hline Heart rate (Mean \pm SD) & & & $<0.001$ \\
Mean & $80.64 \pm 9.59$ & $85.1 \pm 8.29$ & 0.177 \\
Maximum & $146.72 \pm 16.16$ & $143.25 \pm 13.15$ & 0.002 \\
Minimum & $50.47 \pm 6.86$ & $52.63 \pm 6.63$ & $<0.001$ \\
Mean NN (Total), msec Mean \pm SD & $754.88 \pm 90.65$ & $712.76 \pm 69.41$ & 0.002 \\
Mean NN (Daytime), msec Median (IQR) & $710(152)$ & $671(94)$ & $<0.001$ \\
\hline Mean NN (Nighttime), msec Mean \pm SD & $839.64 \pm 144.83$ & $762.42 \pm 91.77$ & \\
\hline
\end{tabular}

*Bpm: beat per minute, SD: standard deviation, NN: NN value is a time between 2 consecutive hearts beats, msec: millisecond. 
Table 3. Comparison of heart rate variability parameters at rest and shift days groups.

\begin{tabular}{|c|c|c|c|}
\hline HRV parameters & Rest & Shift & $\mathrm{p}$ \\
\hline SDNN, msec (Median (IQR)) & $146(57)$ & $116(44)$ & $<0.001$ \\
\hline SDANN, msec (Median (IQR)) & $130(50)$ & $104(43)$ & $<0.001$ \\
\hline rMSSD, msec (Median (IQR)) & $36(20)$ & $31(21)$ & $<0.001$ \\
\hline pNN50, \% (Median (IQR)) & $11.72(16.98)$ & $8.47(13.8)$ & 0.002 \\
\hline TP, msec² (Median (IQR)) & $3000(2788)$ & $2757(2618)$ & 0.04 \\
\hline VLF, msec² (Median (IQR)) & $1471(1063)$ & $1276(1042)$ & 0.017 \\
\hline LF, msec ${ }^{2}$ (Median (IQR)) & $960(828)$ & $873(976)$ & 0.963 \\
\hline HF, msec $^{2}$ (Median (IQR)) & $455(617)$ & $320(549)$ & 0.001 \\
\hline LFnu (Mean \pm SD) & $64.44 \pm 13.82$ & $70.06 \pm 12.35$ & $<0.001$ \\
\hline HFnu (Mean \pm SD) & $35.59 \pm 13.79$ & $29.93 \pm 12.35$ & $<0.001$ \\
\hline LF/HF (Median (IQR)) & $1.89(1.59)$ & $2.51(2.51)$ & $<0.001$ \\
\hline
\end{tabular}

*Bpm: beat per minute, SD: standard deviation, NN: NN value is a time between 2 consecutive hearts beats, msec: millisecond.

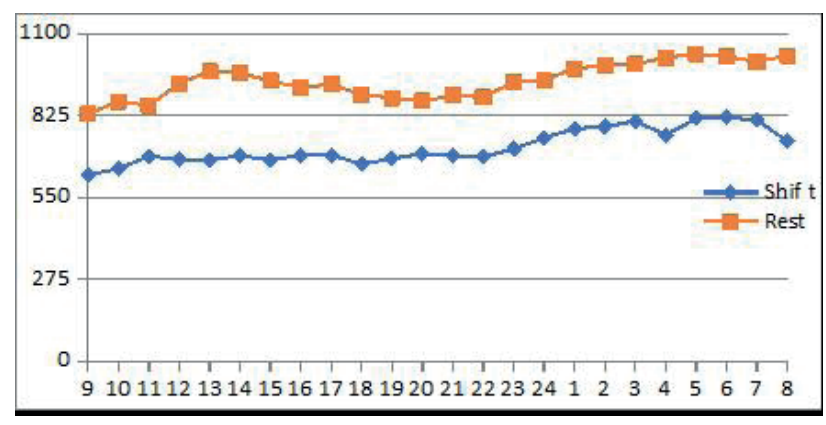

Figure 1. 24-hour trend of average NN value of the groups

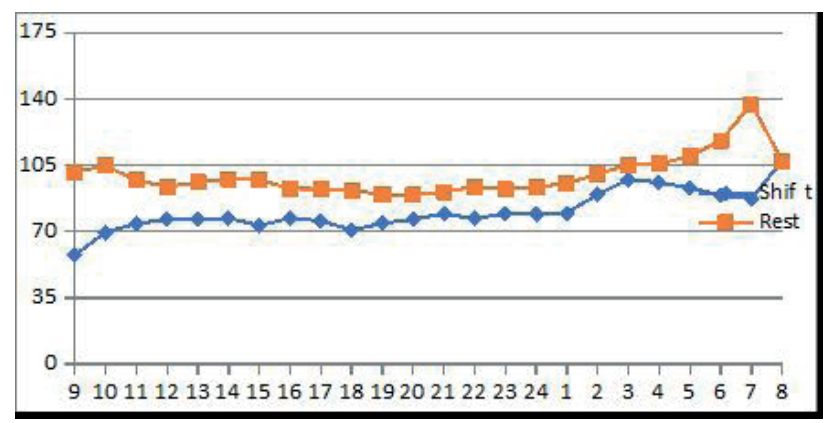

Figure 2. 24-hour trend of mean SDNN value of the groups

Around 3 to 5 o'clock, HFnu parameter during working day showed sudden decrease whereas LFnu parameter showed slight increase in the same time period (Figure 3, Figure 4).

LF/HF ratio showed sudden rise between 03:0005:00 o'clock in shift days (Figure 5).

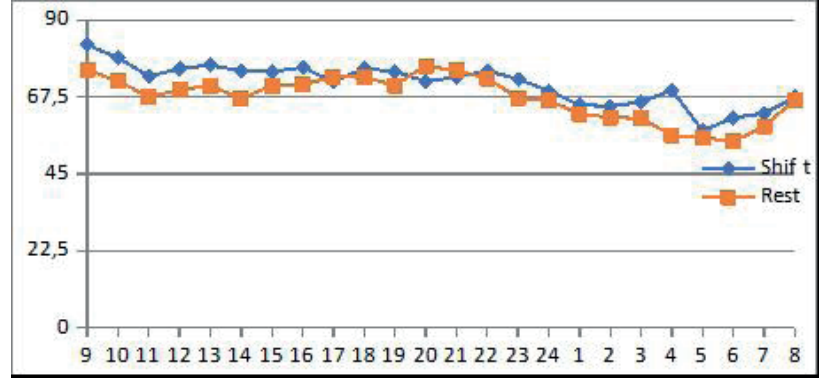

Figure 3. 24-hour trend of average LFnu value of the groups

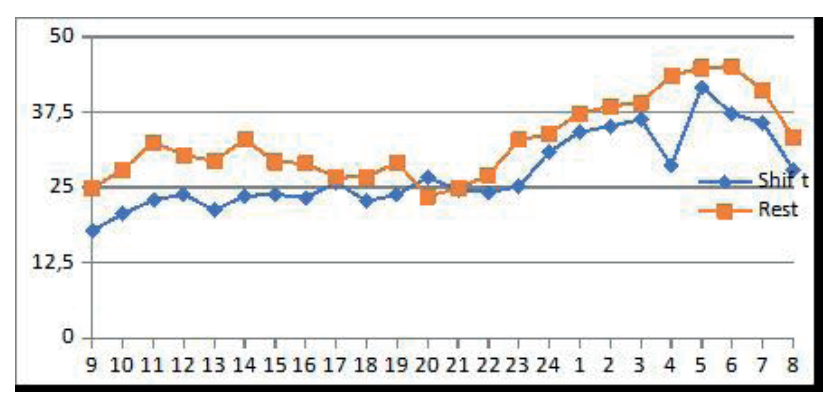

Figure 4. 24-hour trend of average HFnu value of the groups

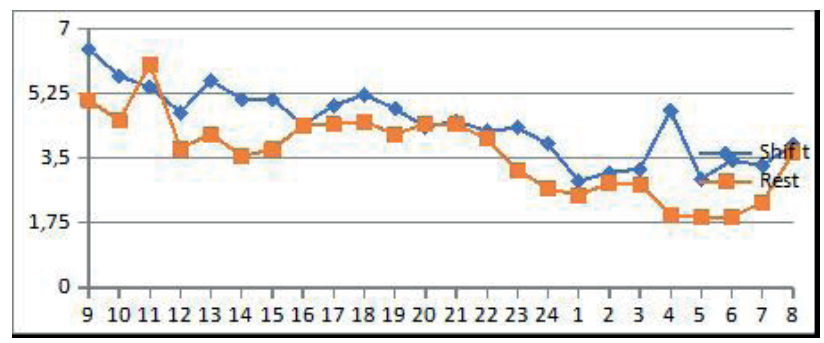

Figure 5. 24-hour trend of mean LF/HF ratio value of the groups 


\section{DISCUSSION}

Heart rate variability is affected by many conditions. These are age, gender, race, physical condition, clinical condition, smoking, drug use, stress and circadian rhythm [14].

Mean and minimum HR values were higher and statistically significant in shift days. This might be explained by increased sympathetic system activation due to stressful working conditions and physical fatigue. Mean NN, day NN and night NN values of rest days were lower than shift days and statistically significant compared to shift days. We explained this situation with the increased stress and workload that force the heart to beat faster in shift days.

In our study, low mean SDNN and SDANN values in shift days were attributed to actively nurse working. The values of rMSSD, VLF, HF and HFnu are affected by the parasympathetic system. In our study, at shift days, significantly lower value of these parameters could be explained by the suppressed parasympathetic system during stressful working time. pNN50 and TP values show heart rate variability directly. If any of the sympathetic or parasympathetic system is dominant, a drop in these values occurs. In the present study, these values were significantly higher in rest days and this was attributed the two systems' concurrent activity. However, lower value of these parameters in shift days were attributed to sympathetic dominance. LFnu and LF/HF ratio are the parameters that show sympathetic dominance very well. High values of these parameters in shift days were attributed to sympathetic dominance as consequences of sleepless and stressors (Table 2).

As comparison of daytime HRV parameters, the values of SDNN, SDANN, rMSSD, pNN50, TP, HF and HFnu were found statistically significant and higher in rest days, however LFnu and LF/HF ratio were statistically significant and higher in shift days. These results were explained by increased activity of the sympathetic system due to high workload and mental fatigue in shift days in opposite to rest days.

When the HRV parameters were compared at night time, rMMSD, pNN50 and HFnu values were statistically significant and higher in rest days, however LFnu and LF/HF ratio were statistically significant and higher in shift days. At shift nights, the physical-mental load caused by insomnia and the stressful conditions played an important role for sympathetic system dominance.

The major finding of our study was that mean NN interval and HRV variables generally showed similar pattern with circadian rhythm irrespective of the working status of the participants. Generally, in both groups, LFnu and LF/HF spectral parameters were higher during the day than during the night whereas vice versa was valid for HFnu parameter. This implies that cardiac autonomic regulation is under the influence of internal clock irrespective of sleep-wakefulness cycle in contrast to a study performed by Ito et al. [12]. They investigated the HRV of nurses during different shifts. They reported that circadian rhythm was determined by physical activity altered by shift work. In another study was performed by Sliskovic et al. [13]. The authors found that HRV was under the influence of pre-set circadian rhythm irrespective of activity. They postulated that the body was not capable of working during night period because of dominancy of parasympathetic activity determined by pre-set circadian rhythm. In our study, we found that HFnu which was a representative of parasympathetic activity exhibited steep decrease between 3-5 o'clock. (Figure 3 and 4). On the other hand, LF nu parameter which reflects sympathetic activity showed slight increase during shift days between 3-5 o'clock. These changes reflect slight increase in sympathetic activity and evident decrease in parasympathetic activity between 3 to 5 o'clock. Vagal tone depression was more prominent within this time period. This peak of sympathetic activity at that time period might be due to early increase for cortisol release to supply the demand of the body to cope with the stress. Circadian rhythm is determined by endogenous hormones, mainly cortisol, controlled by the suprachiasmatic nucleus of the brain [15]. Cortisol level peaks normally during the morning hours (07.00-08.00) [14]. However, this peak may be seen at earlier hours for the night time workers due to sleep disorder [14, 15]. In the present study, between $07.00-08.00$ hours, average NN values showed a minimal increase at the day of rest, but decreased at the day of shift. This situation was attributed to the early peak secretion of cortisol hormone due to corrupted circadian rhythm after working night (Figure 1). 
$\mathrm{LF} / \mathrm{HF}$ ratio is a value that showed similar results as LFnu. These parameters reflect the sympathetic activation. In our study, LF/HF ratio showed similar pattern as LFnu value as expected (Figure 5).

\section{CONCLUSION}

Heart rate variability (HRV) is a simple and noninvasive method of autonomic modulation of the heart [7-9]. HRV measures the of beat to beat fluctuations of the R-R intervals. In shift days, sympathetic dominance was more prominent than resting days. No significant difference was seen in circadian rhythm between the shift and rest days. HRV parameters generally showed similar pattern with circadian rhythm irrespective of the working status. Sympathetic activity was significantly higher in the day period than the night period in the shifting group however there was no difference within resting group. Slight increase in sympathetic system activity and marked decrease in parasympathetic activity during 03-05 o'clock were apparent in shift day group and reflecting possible early release of cortisol. Similar results were found in another study [16]. Borchini et al. found that, prolonged working time and stress exposure were found to delay recovery at the day of rest [17]. In the light of our results, we suggest that nurses should work in short shifts rather than 24-hour shifts.

\section{Limitations}

In our study, we did not measure the relationship between perceived stress evaluated by a questionnaire and HRV parameters.

Another limitation was the relatively small sample size. But we specifically investigated the nurses of coronary care unit. That was the reason of small sample size.

Blood sampling for stress hormone levels during day and night period might add valuable information about the stress level of the participants. Also, this might explain the increase in sympathetic system and decrease in parasympathetic activity during time period of 3 to 5 o'clock.

\section{ACKNOWLEDGEMENTS}

The authors of this paper have no including specific financial interests, relationships, and/or affiliations relevant to the subject matter or materials included. There is no role of the funding source.

\section{CONFLICT Of INTEREST STATEMENT}

The authors of this paper have no competing interests'. 
[1] Knutsson A, Jonsson B, Akerstedt T, et al. Increased risk of ischaemic heart disease in shift workers. The Lancet 1986; 2(8498): 89-92.

[2] Kawachi I, Colditz GA, Stampfer MJ, et al. Prospective study of shift work and risk of coronary heart disease in women. Circulation 1995; 92(11): 3178-82.

[3] Bøggild $H$, Knutsson A. Shift work, risk factors and cardiovascular disease. Scandinavian journal of work, environment \& health. 1999; 25(2): 85-99.

[4] Ferrario MM, Veronesi G, Bertù L, et al. Job strain and the incidence of coronary heart diseases: does the association differ among occupational classes? A contribution from a pooled analysis of Northern Italian cohorts. BMJ open. 2017; 7(1): e014119.

[5] Dragano N, Siegrist J, Nyberg ST, et al. Effort-reward imbalance at work and incident coronary heart disease: a multicohort study of 90,164 individuals. Epidemiology (Cambridge, Mass.). 2017; 28(4): 619-26.

[6] Vrijkotte TG, Van Doornen LJ, De Geus EJ. Effects of work stress on ambulatory blood pressure, heart rate, and heart rate variability. Hypertension 2000; 35(4): 880-6.

[7] Ha M, Kım J, Park J, et al. Blood pressure and heart rate variability in workers of 8-hour shifts. Journal of Human Ergology 2001; 30(1-2): 229-33.

[8] Berntson GG, Thomas Bigger Jr J, Eckberg DL, et al. Heart rate variability: origins, methods, and interpretive caveats. Psychophysiology 1997; 34: 6623-48.
[9] Cardiology ESo. the North American Society of Pacing and Electrophysiology. Heart rate variability: standards of measurement, physiological interpretation, and clinical use; Task Force of the European Society of Cardiology and the North American Society of Pacing and Electrophysiology. Circulation 1996; 93(5): 1043-65.

[10] Stein PK, Kleiger RE. Insights from the study of heart rate variability. Annu Rev Med 1999; 50: 249-61.

[11] Malik M, Camm AJ. Components of heart rate variability-what they really mean and what we really measure. The American journal of cardiology 1993;72(11): 821-2.

[12] Ito $H$, Nozaki $M$, Maruyama T, et al. Shift work modifies the circadian patterns of heart rate variability in nurses. International journal of cardiology 2001; 79(2-): 3231-6.

[13] Slišković A, Manenica I. Changes in heart rate variability during working and non-working nights. Medica Jadertina 2011; 41(1-2): 15-22.

[14] Gamble KL, Berry R, Frank SJ, et al. Circadian clock control of endocrine factors. Nature Reviews Endocrinology 2014; 10(8): 466-75.

[15] Ueyama T, Krout KE, Van Nguyen X, et al. Suprachiasmatic nucleus: a central autonomic clock. Nature neuroscience 1999; 2(12): 1051-3.

[16] Furlan R, Barbic F, Piazza S, et al. Modifications of cardiac autonomic profile associated with a shift schedule of work. Circulation 2000; 102(16): 1912-6.

[17] Borchini R, Veronesi G, Bonzini M, et al. Heart rate variability frequency domain alterations among healthy nurses exposed to prolonged work stress. International journal of environmental research and public health 2018;15(1): 113. 